\title{
Les médecins neuchâtelois dénoncent l'attitude incompréhensible du Groupe Mutuel
}

\author{
Les médecins membres du réseau de soins neuchâtelois (RSN) dénoncent \\ I'attitude incompréhensible du Groupe Mutuel qui rompt ses engagements en \\ faveur de la création d'un réseau de soins de type «managed care» dans le \\ canton de Neuchâtel, ceci après plus d'une année de négociation.
}

\section{Albin Tzaut, Joël Rilliot}

Membres du comité directeur du réseau de soins neuchâtelois
Correspondance: Dr Albin Tzaut Rue A.-Ribaux 11 CH-2022 Bevaix Tél. 0328462822

albin.tzaut@hin.ch
Depuis environ une année, un grand nombre de médecins de premier recours neuchâtelois se sont mobilisés pour mettre en place un réseau de soins de type «managed care» dans le canton. L'objectif de ce type de projet vise à améliorer la coordination des soins entre prestataires de santé (médecins, pharmaciens, physiothérapeutes, etc.), soutenir la formation continue des médecins, optimiser la prise en charge des patients, tout en veillant à garantir le maintien de la qualité des soins et une accessibilité à tous. Le but est également de ralentir l'évolution des coûts de la santé et d'offrir un rabais sur les primes.

Ce type de modèle de prise en charge existe déjà dans de nombreux cantons, parfois depuis plusieurs années et l'expérience montre une excellente efficacité en termes de qualité de la prise en charge tout en permettant des économies. Le travail en réseau implique une collaboration avec les assureurs maladie, notamment pour connaître de façon transparente l'ensemble des coûts engendrés par les différents prestataires de soins, ceci pour mieux analyser les domaines d'actions possibles. Ces coûts globaux restent pour l'heure une propriété exclusive des assureurs.

Jusqu'à présent, les principaux assureurs maladie ont très nettement appuyé la promotion des modèles de soins de type «managed care».

Dans un premier temps, le Groupe Mutuel a manifesté un empressement tout particulier pour encourager la création du réseau et y parti- ciper activement. Des négociations de plus d'une année entre le RSN et le Groupe Mutuel auraient dû aboutir à la conclusion d'un contrat de partenariat. Au dernier instant, le Groupe Mutuel a effectué une volte-face incompréhensible, remettant subitement en doute l'intérêt et l'efficacité des réseaux «managed care» qu'il a pourtant cherché à promouvoir jusque là avec une grande énergie.

Ce revirement semble malheureusement moins surprenant puisqu'il survient peu après l'annonce des propositions de santésuisse d'un modèle d'assurance alternatif qui laisse entrevoir la suppression de l'obligation de contracter. Ce dernier modèle permettrait un règne sans partage des assureurs dans le domaine des soins et reléguerait les prestataires de soins au rang d'exécutants soumis, sans pouvoir décisionnel.

Ce changement radical d'orientation de la part d'un des principaux assureurs maladie de notre pays nous montre la volonté des assureurs maladie de gérer à leur guise notre système de santé et laisse douter de leur réelle motivation à trouver des solutions véritablement consensuelles. Laisser la gestion du système de santé à un seul groupe d'intérêt peut conduire à une dangereuse détérioration de la qualité des soins.

Le RSN poursuit des négociations avec d'autres assureurs en espérant rencontrer un état d'esprit plus favorable. 